\title{
DISTRIBUTION OF DR-TB BY SEX, AGE GROUPS, OCCUPATION, PROVINCE, DIVISION, DISTRICT, TYPE OF DISEASE, TYPE OF DRUG RESISTANCE, TREATMENT REGIMEN AND OUTCOME OF TREATMENT IN DR-TB POPULATION IN D.I.KHAN DIVISION, PAKISTAN
}

\begin{abstract}
Zeeshan Saleem¹, Imran Ullah², Muhammad Sarim Bin Farooq Awan', Jamal Tauqir', Faisal Younis ${ }^{3}$, Nisar Khan', Huzaifa Tahir Saeed Siddique', Asim Alam¹, Noman Ullah', Muhammad Asif', Muhammad Arif', Sadeeq Ahmad', Hamza Shafiq', Sohail Ahmad Meer', Rahmail Khan', Muhammad Hamza Riaz', Muhammad Ayub ${ }^{1}$

${ }^{1}$ Students, ${ }^{2}$ Department of Health, D.I.Khan, ${ }^{3}$ PMDT Center, MMM Teaching Hospital, D.I.Khan, ${ }^{4}$ Department of Medicine, ${ }^{1,4} \mathrm{Gomal}$ Medical College, D.I.Khan, Pakistan
\end{abstract}

\begin{abstract}
Background: Drug-resistant tuberculosis (DR-TB) is the major cause of mortality worldwide. Our objectives were to determine the distribution of DR-TB by sex, age groups, occupation, province, division, district, type of disease, type of drug resistance, treatment regimen and outcome of treatment in DR-TB population in D.I.Khan Division, Pakistan.

Materials \& Methods: This cross-sectional study was conducted in Department of Community Medicine, Gomal Medical College, D.I.Khan, Pakistan. A sample of 286 DR-TB patients was selected consecutively from population at risk. Sex, age groups, occupation, province, division and district were demographic while type of disease, type of drug resistance, treatment regimen and outcome of treatment were research variables. All variables being nominal were described by count, percentage \& cumulative percentage with $95 \%$ confidence interval for proportion. Distribution of DR-TB patients by all the ten variables were substantiated by chi-square goodness-of-fit test.

Results: Out of 286 DR-TB patients, $123(43 \%)$ were men and $163(57 \%)$ women. DR-TB cases were most prevalent in age group 15-44 years 172 (60.14\%), housewife 140 (48.95\%), Khyber Pakhtunkhwa 175 (61.19\%), D.I.Khan Division 178 (62.24\%) and district 121 (42.31\%). Most common type of disease, drug resistance and treatment regimen was pulmonary TB $282(98.60 \%)$, MDR $273(95.45 \%)$ and longer treatment ( $n=273$ MDR-TB) $246(90.11 \%)$ respectively. Treatment success rate was 161 (56.29\%). The observed prevalence by occupation, province, division, district and type of disease in our sample was similar to expected prevalence in population ( $p>.05$ for all), while it was different from population by sex, age groups, type of drug resistance, regimen and treatment outcome $(p<.05$ for all).

Conclusion: The prevalence of DR-TB was higher in women, age group 15-44 years, housewife, Khyber Pakhtunkhwa and D.I.Khan Division and District. Most common type of disease, drug resistance and treatment regimen was pulmonary TB, MDR and longer treatment respectively. Treatment success rate was $56.29 \%$. The observed prevalence by occupation, province, division, district and type of disease in sample was similar to population, while it was different by sex, age groups, type of drug resistance, regimen and treatment outcome.

KEY WORDS: Tuberculosis; Pulmonary Tuberculosis; Drug-Resistant Tuberculosis, Multidrug-Resistant Tuberculosis; MDR Tuberculosis; Extensively Drug-Resistant Tuberculosis; XDR-TB; Drug Resistance; Multidrug Resistance.
\end{abstract}

Cite as: Saleem Z, Ullah I, Younis F, Khan N, Awan MSBF, Tauqir J, et al. Distribution of DR-TB by sex, age groups, occupation, province, division, district, type of disease, type of drug resistance, treatment regimen and outcome of treatment in DR-TB population in D.I.Khan Division, Pakistan. Gomal J Med Sci 2020 Jul-Sep; 18 (3):115-31. https://doi.org/10.46903/gjms/18.03.897

Corresponding Author:

Zeeshan Saleem

Student, Gomal Medical College

D.I.Khan, Pakistan

E-mail: zeeshan.saleem02@gmail.com

Date Submitted: 01-06-2020

Date Revised: 22-06-2020

Date Accepted:

\section{INTRODUCTION}

1.1 Background Tuberculosis (TB) is the $10^{\text {th }}$ leading cause of mortality worldwide and the leading cause of death from a single infectious disease. Globally in 2018, about 10 million people suffered from TB. TB causes 1.2 million deaths in HIV non-infected patients and 251,000 deaths in HIV infected patients. 
In 2018 the highest prevalence was in adult men $57 \%$, followed by adult women $32 \%$ and children $11 \%$. There was $8.6 \%$ prevalence of TB in HIV patients. Eight countries are responsible for two thirds of the global TB burden: India $27 \%$, Indonesia $8 \%$, China 9\%, Philippines 6\%, Pakistan 6\%, Nigeria 4\%, Bangladesh $4 \%$ and South Africa 3\%. Globally in 2018 , about $3.4 \%$ new TB cases and $18 \%$ previously treated cases had MDR/RR TB. In 2018, about 0.5 million incident cases were rifampicin-resistant (RR); out of which $78 \%$ were multidrug-resistant (MDR) TB. About 214,000 deaths occurred from MDR-TB in $2017 .^{1}$

Pakistan is $5^{\text {th }}$ high-prevalent country for TB and $5^{\text {th }}$ for DR-TB. Estimated TB incidence, prevalence and mortality is $267 / 100,000,341 / 100,000$ and $27 / 100,000$ population respectively with about 525,000 annual incident TB cases. ${ }^{2}$

Worku, et al. ${ }^{3}$ from Addis Ababa, Ethiopia from November 2011 to December 2016 with 340 pulmonary MDR/RR-TB cases notified distribution as 180 (52.94\%) men and 160 (47.06\%) women, 5 (1.5\%) in age group <15 years, $105(30.9 \%)$ in 15-24, 199 (58.5\%) in $25-44$ and 31 (9.1\%) in $\geq 45$ years.

Johnston, et al. ${ }^{4}$ from Canada reported successful treatment in $62 \%$ (95\% Cl 57-67), death in 11\% (95\% $\mathrm{Cl} 9-13)$, failed therapy in $8 \%(95 \% \mathrm{Cl} 5-11)$, default rate $13 \%(95 \% \mathrm{Cl} 9-17)$ and $2 \%(95 \% \mathrm{Cl} 1-4)$ were not evaluated out of 4,959 MDR-TB patients for years 1973-2006.

$\mathrm{He}$, et al. ${ }^{5}$ from Shandong Province, China from January 2007 to December 2014 reported 13,486 culture-confirmed TB cases, of which $21.2 \%(2,855 * 100 / 13,486=21.2 \%)$ were resistant to at least one first line anti-TB drugs, $6.2 \%$ $(838 * 100 / 13,486=6.2 \%)$ were MDR-TB patients and $1.2 \%\left(16^{*} 100 / 13,486=1.2 \%\right)$ were resistant to rifampicin.

Alene, et al. ${ }^{6}$ from Changsha, Hunan, China from 2011 to 2014 reported 481 DR-TB, including 471 (98\%) MDR-TB and 10 (2\%) XDR-TB. Out of 481 DRTB cases, 340 (70.7\%) were men and 141 (29.3\%) women, 380 (79.0\%) farmer, 15 (3.1\%) labourer, 16 (3.3\%) employed, 19 (3.8\%) unemployed and 51 (10.6\%) other/unknown, 261 (54.3\%) were cured, 14 (2.9\%) completed, 13 (2.7\%) died, 63 (13.1\%) failure and $130(27.0 \%)$ loss to follow up cases.

Venkatesh, et al. ${ }^{7}$ from Gorakhpur, India from November 2015, to October 2016 reported 157 MDR-TB patients as $80(51 \%, 95 \% \mathrm{Cl} 43.2-58.6)$ in age group $18-29$ years, $45(28.7 \%, 95 \% \mathrm{Cl} 22.1-36.1)$ in $30-40$ and $32(20.3 \% 95 \% \mathrm{Cl} 14.8-27.3)$ in $>40$ years, 108 $(68.8 \%, 95 \% \mathrm{Cl} 61.1-75.5)$ in men and $49(31.2 \%$, $95 \% \mathrm{Cl} 24.4-38.8)$ in women and $153(97.5 \%, 95 \% \mathrm{Cl}$ 93.6-99.0) had pulmonary TB, $2(1.3 \%, 95 \% \mathrm{Cl} 0.3-4.5)$ had pulmonary \& pleural TB and $2(1.3 \% 95 \%$ Cl $0.3-$
4.5) had pulmonary \& other system TB respectively. Hasan, et al. ${ }^{8}$ from Karachi, Pakistan from 2006 to 2009 notified frequency of MDR and XDR TB among 9,523 mycobacterium tuberculosis isolates as $38.66 \%(3,682 * 100 / 9,523=38.66 \%)$ and $1.19 \%$ $(113 * 100 / 9,523=1.19 \%)$ respectively.

Akhter, et al. ${ }^{9}$ from Lahore, Pakistan from Jan. 2010 to June 2014 distributed 1,250 retreatment DR-TB cases across MDR-TB and mono/ poly-resistant TB as $861(69 \%)$ and 389 (31\%), across men and women as 664 (53.12\%) and 586 (46.88\%), across age groups of 0-14 years, $15-45$ and $>46$ years as 31 (2.48\%), 996 (79.68\%) and 223 (17.84\%) and across pulmonary and extra pulmonary TB as 1231 (98.48\%) and 19 (1.52\%) respectively.

Wahab, et al. ${ }^{10}$ from Peshawar, Pakistan from December 2006 to October 2007 showed distribution of 30 MDR-TB cases as $17(56.7 \%)$ men and 13 $(43.3 \%)$ women, $4(13.3 \%)$ in age group $<20$ years, $18(60 \%)$ in $20-40,8(26.7 \%)$ in $>40$ years, $11(36.67$ \%) labourer, 1 (3.33\%) student, 2 (6.67 \%) govt. employee, 13 (43.33\%) housewife and 3 (10\%) others.

Ahmad, et al. ${ }^{11}$ from Peshawar, Pakistan from Jan. 2012 to July 2013 reported 289 DR-TB cases. Out of 289 DR-TB cases, 17 (5.88\%) were treated MDRTB cases, 243 (84.08\%) newly diagnosed MDR-TB cases (total MDR-TB cases $17+243=260(89.96 \%)$, $10(3.46 \%)$ were mono-resistant TB, six $(2.08 \%)$ poly-resistant TB and $13(4.50 \%)$ XDR-TB. Out of 243 newly diagnosed MDR-TB, 238 (97.9\%) were pulmonary, two $(0.8 \%)$ pulmonary \& extra pulmonary (total pulmonary cases $238+2=240$ [98.77\%]) and three $(1.23 \%)$ extra-pulmonary cases.

Arshad, et al. ${ }^{12}$ from Peshawar, Pakistan from February 2011 to December 2012 reported $51.76 \%$ $(132 * 100 / 255=51.76)$ MDR-TB cases out of 255 DR-TB cases. Out of 132 MDR-TB cases, 67 $(67 * 100 / 132=50.76 \%)$ were eligible for shorter treatment regimen, while $65(65 * 100 / 132=49.24 \%)$ were not eligible.

\subsection{Research Problems (RPs), Knowledge Gaps (KGs) \& Justification}

Allocation of resources is planned on the basis of overall and group wise burden of the problem/ disease. Unawareness of distribution of drug resistant tuberculosis (DR-TB) by sex, age groups, occupation, province, division, district, type of disease, type of drug resistance, treatment regimen and outcome of treatment in DR-TB population in D.I.Khan Division, Pakistan are our ten RPs. Unavailability of data regarding these RPs on various databases like Google Scholar, PubMed, ScienceDirect, ProQuest and Pakistan Research Repository are our ten KGs. To fill these KGs is justification of our project. 


\subsection{Research Questions (RQs)}

$R Q$ 1-10: What is the distribution of DR-TB by sex, age groups, occupation, province, division, district, type of disease, type of drug resistance, treatment regimen and outcome of treatment respectively in DR-TB population in D.I.Khan Division, Pakistan?

$R Q$ 11-20: Is there any difference between the observed and expected distribution of DR-TB by sex, age groups, occupation, province, division, district, type of disease, type of drug resistance, treatment regimen and outcome of treatment respectively in DR-TB population in D.I.Khan Division, Pakistan?

\subsection{Research Objectives (ROs)}

The objectives of our study were to determine the:

RO 1-10: distribution of DR-TB by sex, age groups, occupation, province, division, district, type of disease, type of drug resistance, treatment regimen and outcome of treatment respectively in DR-TB population in D.I.Khan Division, Pakistan.

RO 11-20: difference between the observed and expected distribution of DR-TB by sex, age groups, occupation, province, division, district, type of disease, type of drug resistance, treatment regimen and outcome of treatment respectively in DR-TB population in D.I.Khan Division, Pakistan.

\subsection{Research Hypotheses (Null)}

$\mathbf{H}_{01}$ : There is no difference between the observed and expected distribution of DR-TB by sex in DRTB population in D.I.Khan Division, Pakistan. (RQ 11, RO 11)

$\mathbf{H}_{02}$ : There is no difference between the observed and expected distribution of DR-TB by age groups in DR-TB population in D.I.Khan Division, Pakistan. (RQ 12, RO 12)

$\mathbf{H}_{03}$ : There is no difference between the observed and expected distribution of DR-TB by occupation in DR-TB population in D.I.Khan Division, Pakistan. (RQ 13, RO 13)

$\mathbf{H}_{04}$ : There is no difference between the observed and expected distribution of DR-TB by province in DR-TB population in D.I.Khan Division, Pakistan. (RQ 14, RO 14)

$\mathbf{H}_{05}$ : There is no difference between the observed and expected distribution of DR-TB by division in DR-TB population in D.I.Khan Division, Pakistan. (RQ 15, RO 15)

$H_{06}$ : There is no difference between the observed and expected distribution of DR-TB by district in DR-TB population in D.I.Khan Division, Pakistan. (RQ 16, RO 16)

$H_{07}$ : There is no difference between the observed and expected distribution of DR-TB by type of disease in DR-TB population in D.I.Khan Division, Pakistan. (RQ 17, RO 17)
$\mathbf{H}_{08}$ : There is no difference between the observed and expected distribution of DR-TB by type of drug resistance in DR-TB population in D.I.Khan Division, Pakistan. (RQ 18, RO 18)

$\mathbf{H}_{09}$ : There is no difference between the observed and expected distribution of DR-TB by treatment regimen in DR-TB population in D.I.Khan Division, Pakistan. (RQ 19, RO 19)

$\mathbf{H}_{10}$ : There is no difference between the observed and expected distribution of DR-TB by outcome of treatment in DR-TB population in D.I.Khan Division, Pakistan. (RQ 20, RO 20)

\subsection{Operational definitions ${ }^{13}$}

Mono-resistance: "Resistance to any one first-line anti-TB drug only".

Poly-resistance: "Resistance to more than one first-line anti-TB drugs, other than both isoniazid and rifampicin".

Multidrug resistance (MDR): "Resistance to at least both isoniazid and rifampicin".

Extensive drug resistance (XDR): "Resistance to any fluoroquinolone, and at least one of three second-line injectable drugs (capreomycin, kanamycin and amikacin), in addition to multidrug resistance".

Rifampicin resistance (RR): "Resistance to rifampicin detected using phenotypic or genotypic methods, with or without resistance to other anti-TB drugs. It includes any resistance to rifampicin, in the form of mono-resistance, poly-resistance, MDR or XDR".

Multi drug-resistance TB (MDR-TB): "Resistance to at least both isoniazid and rifampicin".

Extensive drug-resistance TB (XDR-TB): "Resistance to any fluoroquinolone $F Q$ and at least one of the injectable second-line drugs SLIs (amikacin or streptomycin) in addition to multidrug resistance".

Cured: "Treatment completed as recommended by the national policy (minimum 18 months past culture conversion) without evidence of failure and three or more consecutive cultures taken at least 30 days apart are negative after the intensive phase".

Treatment completed: "Treatment completed as recommended by the national policy (minimum 18 months past culture conversion) without evidence of failure but no record that three consecutive cultures taken at least 30 days apart are negative after the intensive phase".

Failure: "Treatment terminated or need for permanent regimen change of at least two anti-TB drugs because of:

- lack of conversion by the end of the intensive phase, or

- bacteriological reversion in the continuation phase after conversion to negative, or 
- evidence of additional acquired resistance to fluoroquinolone or second-line injectable drugs, or

- adverse drug reactions (ADRS)"

Died: "A patient who dies for any reason during the course of treatment".

Lost to follow-up: "A patient whose treatment was interrupted for two consecutive months or more".

Not evaluated: "A patient for whom no treatment outcome is assigned (this included cases "transferred out" to another treatment unit and whose treatment outcome is unknown)".

\section{MATERIALS AND METHODS}

2.1 Design, Setting \&Duration: This crosssectional study was conducted at the Department of Community Medicine, Gomal Medical College, D.I.Khan, Pakistan from January 10, 2020 to February16, 2020. The proposal for this project was approved by the Institutional Research \& Ethics Committee. The project was supervised by Dr. Muhammad Marwat.

2.2 Population, Sample Size, Technique and Selection: Programmatic Management Unit of Drug Resistant Tuberculosis (PMDT) Center of our hospital provides health services for DR-TB to D.I.Khan Division, which consists of D.I.Khan \& Tank districts, South Waziristan Agency \& Frontier Region of Darazinda. The population of the Division was $2,803,147$ in 2017 Census. The prevalence of TB in Pakistan is estimated for the year 2017 as $341 / 100,000$ population. ${ }^{1}$ DR-TB would contribute its $5 \%(5 * 341 / 100=17.05)$ as $17.05 / 100,000$ $(17.05 * 100 / 100,000=0.017 \%){ }^{2}$

Using Raosoft ${ }^{14}$ online calculator, with margin of error $0.1511 \%$, confidence level $95 \%$, population size at risk for DR-TB 2,803,147 and assumed prevalence of $0.017 \%$ of DR-TB in this population, sample size of 286 was calculated.

All patients of DR-TB were eligible for inclusion with no exclusion criteria.

\subsection{Procedure of Conduct}

The data of 286 DR-TB patients was retrieved from ENRS (Electronic Nominal Registration System) maintained at PMDT Center, MMM Teaching Hospital, D.I.Khan, Pakistan. This PMDT Center was established on September 01, 2013 and first patient was registered on October 29, 2013. These 286 patients were registered till December 31, 2019, a data set for six years.

\subsection{Data Collection Plan}

Secondary was collected through literature search, while primary data was collected through observation. Primary data was collected for the following six demographic variables (attributes); sex (men, women), age group (0-14 years, 15-44 and $\geq 45$ years), occupation (office worker, labourer \& housewife), province (Khyber Pakhtunkhwa, Punjab, Baluchistan, Sindh \& FATA), division (D.I.Khan, Bannu, Sargodha, Zhob and Others) and district (D.I.Khan, Tank, Lakki Marwat, Bannu, Bhakkar, Mianwali, Zhob, South Waziristan Agency, North Waziristan Agency \& others).

Data was collected for the following four research variables (attributes); type of disease (pulmonary, extra-pulmonary), type of drug resistance (mono-resistance, poly-resistance, MDR \& XDR), treatment regimen (shorter treatment regimen, longer treatment regimen) and outcome of treatment (cured, treatment completed, died, failed, lost to follow up, not evaluated \& still under treatment). All these variables were measured on nominal scale except age groups on ordinal scale.

\subsection{Data Analysis Plan}

\subsubsection{Descriptive Analysis and Estimation of $\mathrm{Pa}$ - rameters}

Descriptively all the ten variables were analyzed by count, percentage and cumulative percentage. Estimation of parameters for proportion for the population was given as confidence interval at confidence level of $95 \%$, using Wilson score interval for the binomial distribution through an online statistical calculator. ${ }^{15} \mathrm{It}$ would mean that if the confidence interval is calculated for an infinite number of samples with a sample size of $286,95 \%$ of the calculated confidence intervals will contain the true value of the proportion of the population.

\subsubsection{Testing of Hypotheses}

Distribution of DR-TB by sex, age groups, occupation, province, division, district, type of disease, type of drug resistance, treatment regimen and outcome of treatment were analyzed separately by chi-square goodness-of-fit test $\left(\mathrm{H}_{01}-\mathrm{H}_{10}\right){ }^{16-17}$ Observed counts, expected counts, their difference, chi-square value, degree of freedom and level of significance were given at alpha .05 by an online calculator. ${ }^{18,19}$

\section{RESULTS}

\subsection{Descriptive Analysis and Estimation of Param-} eters

\subsubsection{Distribution of DR-TB by sex (RQ 1)}

Out of 286 patients with DR-TB, $123(43 \%, 95 \% \mathrm{Cl}$ $37.39-48.80)$ were men and 163 (57\%, 95\% Cl 51.1962.60) women.

\subsubsection{Distribution of DR-TB by age groups (RQ 2)}

The highest prevalence was in age group 15-44 years $172(60.14 \%, 95 \% \mathrm{Cl} 54.37-65.81)$ patients. (Table 3.1.2)

3.1.3 Distribution of DR-TB by occupation (RQ 3) The housewife was the modal occupation category with 140 (48.95\%) patients. (Table 3.1.3) 
3.1.4 Distribution of DR-TB by province (RQ 4)

Khyber Pakhtunkhwa province had highest number of patients as 175 (61.19\%). (Table 3.1.4)

3.1.5 Distribution of DR-TB by Division (RQ 5)

D.I.Khan Division has the highest prevalence of cases 178 (62.24\%). Others included one each from Layyah, Chakwal and Larkana districts. (Table 3.1.5)

3.1.6 Distribution of DR-TB by district (RQ 6)

D.I.Khan district had highest number of patients as 121 (42.31\%). Others included one each from

Table 3.1.2: Distribution of DR-TB by age groups in DR-TB population in D.I.Khan Division, Pakistan $(\mathbf{n}=\mathbf{2 8 6})$

\begin{tabular}{|c|l|c|c|c|c|c|}
\hline \multirow{2}{*}{ Variable } & \multirow{2}{*}{ Attributes } & \multicolumn{3}{|c|}{ Sample Statistics } & \multicolumn{2}{c|}{$95 \%$ Cl for Proportion } \\
\cline { 3 - 7 } & & Count & Percentage & Cumulative \% & Lower & Upper \\
\hline \multirow{3}{*}{ Age Group } & $0-14$ years & 8 & 2.80 & 2.80 & 1.40 & 5.42 \\
\cline { 2 - 7 } & $15-44$ years & 172 & 60.14 & 62.94 & 54.37 & 65.81 \\
\cline { 2 - 7 } & $\geq 45$ years & 106 & 37.06 & 100.00 & 31.67 & 42.79 \\
\hline \multicolumn{2}{|c|}{ Total } & 286 & $100 \%$ & & \multicolumn{2}{c|}{ Population Parameters } \\
\hline
\end{tabular}

Table 3.1.3: Distribution of DR-TB by occupation in DR-TB population in D.I.Khan Division, Pakistan $(\mathbf{n}=\mathbf{2 8 6})$

\begin{tabular}{|c|l|c|c|c|c|c|}
\hline \multirow{2}{*}{ Variables } & \multirow{2}{*}{ Attributes } & \multicolumn{3}{|c|}{ Sample Statistics } & \multicolumn{2}{c|}{$95 \%$ Cl for Proportion } \\
\cline { 3 - 7 } & & Count & Percentage & Cumulative \% & Lower & Upper \\
\hline \multirow{3}{*}{ Occupation } & Office worker & 33 & 11.54 & 11.54 & 08.33 & 15.76 \\
\cline { 2 - 7 } & Labor & 113 & 39.51 & 51.05 & 34.01 & 45.27 \\
\cline { 2 - 7 } & House wife & 140 & 48.95 & $100 \%$ & 43.21 & 54.71 \\
\hline \multicolumn{2}{|c|}{ Total } & 286 & $100 \%$ & & \multicolumn{2}{c|}{ Population Parameters } \\
\hline
\end{tabular}

Table 3.1.4: Distribution of DR-TB by province in DR-TB population in D.I.Khan Division, Pakistan $(\mathbf{n}=\mathbf{2 8 6})$

\begin{tabular}{|l|l|c|c|c|c|c|}
\hline \multirow{2}{*}{ S. No. } & \multirow{2}{*}{ Province } & \multicolumn{3}{|c|}{ Sample Statistics } & \multicolumn{2}{c|}{ 95\% Cl for Proportion } \\
\cline { 3 - 6 } & & Count & Percentage & Cumulative \% & Lower & Upper \\
\hline 1 & Khyber Pakhtunkhwa & 175 & 61.19 & 61.19 & 55.42 & 66.65 \\
\hline 2 & Punjab & 56 & 19.58 & 80.77 & 15.39 & 24.56 \\
\hline 3 & Baluchistan & 9 & 03.15 & 83.92 & 01.66 & 05.87 \\
\hline 4 & Sindh & 1 & 00.35 & 84.27 & 00.06 & 01.95 \\
\hline 5 & FATA & 45 & 15.73 & $100 \%$ & 11.97 & 20.40 \\
\hline \multicolumn{2}{|l|}{ Total } & 286 & $100 \%$ & & \multicolumn{2}{c|}{ Population Parameters } \\
\hline
\end{tabular}

Table 3.1.5: Distribution of DR-TB by division in DR-TB population in D.I.Khan Division, Pakistan $(n=286)$

\begin{tabular}{|l|l|c|c|c|c|c|}
\hline \multirow{2}{*}{ S. No. } & \multirow{2}{*}{ Division } & \multicolumn{3}{|c|}{ Sample Statistics } & \multicolumn{2}{c|}{ 95\% Cl for Proportion } \\
\cline { 3 - 7 } & & Count & Percentage & Cumulative \% & Lower & Upper \\
\hline 1 & D.I.Khan & 178 & 62.24 & 62.24 & 56.49 & 67.66 \\
\hline 2 & Bannu & 40 & 13.99 & 76.23 & 10.45 & 18.49 \\
\hline 3 & Sargodha & 56 & 19.58 & 95.81 & 15.40 & 24.57 \\
\hline 4 & Zhob & 9 & 3.15 & 98.96 & 1.67 & 5.88 \\
\hline 5 & Others & 3 & 1.04 & $100.00 \%$ & 0.35 & 3.02 \\
\hline \multicolumn{2}{|l|}{ Total } & 286 & $100.00 \%$ & & \multicolumn{2}{c}{ Population Parameters } \\
\hline
\end{tabular}


Distribution of DR-TB by sex, age groups, occupation, province, division, district, type of disease, type...

Layyah, Chakwal and Larkana districts. (Table 3.1.6)

3.1.7 Distribution of DR-TB by type of disease (RQ 7)

There were $282(98.60 \%, 95 \% \mathrm{Cl} 96.45-99.45 \%)$ patients of pulmonary and $4(01.40 \%, 95 \% \mathrm{Cl} 0.54-$ $3.54 \%)$ patients of extra-pulmonary tuberculosis.

3.1.8 Distribution of DR-TB by type of drug resistance (RQ 8)

MDR-TB was most prevalent 273 (95.45\%). (Table 3.1.8)
3.1.9 Distribution of MDR-TB by treatment regimen (RQ 9)

Out of 273 MDR-TB cases (273 MDR-TB/286 DR-TB), $27(9.89 \%, 95 \% \mathrm{Cl} 6.89-14.01)$ were on STR, while 246 (90.11\%, 95\% Cl 85.99-93.11) on LTR.

3.1.10 Distribution of DR-TB by outcome of treatment (RQ 10)

The outcome of treatment in term of patients cured was 158 (55.24\%). (Table 3.1.10)

Table 3.1.6: Distribution of DR-TB by district in DR-TB population in D.I.Khan Division, Pakistan $(\mathbf{n}=\mathbf{2 8 6})$

\begin{tabular}{|l|l|c|c|c|c|c|}
\hline \multirow{2}{*}{ S. No. } & \multirow{2}{*}{ District } & \multicolumn{3}{|c|}{ Sample Statistics } & \multicolumn{2}{c|}{ 95\% Cl for Proportion } \\
\cline { 3 - 6 } & & Count & Percentage & Cumulative \% & Lower & Upper \\
\hline 1 & D.I.Khan & 121 & 42.31 & 42.31 & 36.70 & 48.10 \\
\hline 2 & Tank & 18 & 06.29 & 48.60 & 04.01 & 09.72 \\
\hline 3 & Lakki Marwat & 27 & 09.44 & 58.04 & 06.56 & 13.39 \\
\hline 4 & Bannu & 9 & 03.15 & 61.19 & 01.66 & 05.87 \\
\hline 5 & Bhakkar & 49 & 17.13 & 78.32 & 13.21 & 21.93 \\
\hline 6 & Mianwali & 5 & 01.75 & 80.08 & 00.74 & 04.02 \\
\hline 7 & Zhob & 9 & 03.15 & 83.22 & 01.66 & 05.87 \\
\hline 8 & South Waziristan Agency & 41 & 14.33 & 97.55 & 10.74 & 18.86 \\
\hline 9 & North Waziristan Agency & 4 & 1.40 & 98.95 & 00.55 & 03.54 \\
\hline 10 & Others & 3 & 1.05 & $100.00 \%$ & 00.36 & 3.04 \\
\hline & Total & 286 & $100.00 \%$ & & \multicolumn{2}{c|}{ Population Parameters } \\
\hline
\end{tabular}

Table 3.1.8: Distribution of DR-TB by type of drug resistance in DR-TB population in D.I.Khan Division, Pakistan $(n=286)$

\begin{tabular}{|l|l|c|c|c|c|c|}
\hline \multirow{2}{*}{ S. No. } & \multirow{2}{*}{ Type of drug resistance } & \multicolumn{3}{|c|}{ Sample Statistics } & \multicolumn{2}{c|}{$95 \%$ Cl for Proportion } \\
\cline { 3 - 7 } & & Count & Percentage & Cumulative \% & Lower & Upper \\
\hline 1 & Mono-drug resistance & 1 & 00.35 & 00.35 & 00.06 & 01.95 \\
\hline 2 & Poly-drug resistance & 2 & 00.70 & 01.05 & 00.19 & 02.51 \\
\hline 3 & MDR & 273 & 95.45 & 96.50 & 92.37 & 97.87 \\
\hline 4 & XDR & 10 & 03.50 & $100.00 \%$ & 01.91 & 05.63 \\
\hline \multicolumn{2}{|c|}{ Total } & 286 & $100.00 \%$ & & \multicolumn{2}{c|}{ Population Parameters } \\
\hline
\end{tabular}

Table 3.1.10: Distribution of DR-TB by outcome of treatment in DR-TB population in D.I.Khan Division, Pakistan $(n=286)$

\begin{tabular}{|l|l|c|c|c|c|c|}
\hline \multirow{2}{*}{ S. No. } & \multirow{2}{*}{ Outcome of Treatment } & \multicolumn{3}{|c|}{ Sample Statistics } & \multicolumn{2}{c|}{ 95\% Cl for Proportion } \\
\cline { 3 - 6 } & & Count & Percentage & Cumulative \% & Lower & Upper \\
\hline 1 & Cured & 158 & 55.24 & 55.24 & 49.45 & 60.90 \\
\hline 2 & Treatment completed & 3 & 01.05 & 56.29 & 00.35 & 03.03 \\
\hline 3 & Died & 59 & 20.63 & 76.92 & 16.34 & 25.69 \\
\hline 4 & Failed & 9 & 03.15 & 80.07 & 01.66 & 05.87 \\
\hline 5 & Lost to follow up & 31 & 10.84 & 90.91 & 07.74 & 14.97 \\
\hline 6 & Not evaluated & 6 & 02.09 & 93.00 & 00.96 & 04.50 \\
\hline 7 & Still under treatment & 20 & 07.00 & 100.00 & 04.57 & 10.55 \\
\hline \multicolumn{2}{|r|}{ Total } & 286 & 100 & & Population Parameters \\
\hline
\end{tabular}




\subsection{Hypotheses Testing}

3.2.1 Distribution of DR-TB by sex (RQ 1 \& 11, $\mathbf{H}_{01}$ ): Our observed distribution for men versus women was 123:163 from a sample of 286 against expected counts of 664:586 from 1,250 previously treated DR-TB cases by Akhter, et al. ${ }^{9}$ These were not comparable due to different sample sizes/ denominators. Hence the expected counts and expected percentages were adjusted for a sample of 286. The expected counts of 664:586 were replaced by 151.92:134.08 (adjusted expected). Adjusted expected percentages came similar to expected percentages, so not changed. (Table 3.2.1.1)

The observed distribution of DR-TB by sex in sample was compared to the expected distribution in the population through chi-square goodness-of-fit test, which showed p-value <alpha. $\mathrm{H}_{01}$ was declared as false and therefore rejected, showing that the observations did not fit the statistical model of the population. In simple words, our observed prevalence of DR-TB in men $43 \%$ was statisti- cally significantly lower to what we expected (adjusted) for men $53.12 \%$ \& our observed prevalence of DR-TB in women $57 \%$ was higher to what we expected (adjusted) for women $46.88 \%$ from Akhter, et al..$^{9}$ (Table 3.2.1.2)

3.2.2 Distribution of DR-TB by age groups (RQ 2 \& 12, $\mathrm{H}_{02}$ ): Our observed distribution for age groups (in years) of 0-14:15-44: $\geq 45$ was 8:172:106 from a sample of 286 against expected counts of 31:996:223 from 1,250 previously treated DR-TB cases by Akhter, et al. ${ }^{9}$ These were not comparable due to different sample sizes/ denominators. Hence the expected counts and expected percentages were adjusted for a sample of 286 . The expected counts of 31:996:223 were replaced by 7.09:227.89:51.02 (adjusted expected). Adjusted expected percentages came similar to expected percentages, so not changed. (Table 3.2.2.1)

The observed distribution of DR-TB by age groups in sample was compared to the expected distribution in the population through chi-square goodness-

Table 3.2.1.1:Observed, expected and adjusted expected counts and percentages for distribution of DR-TB population by sex in D.I.Khan Division, Pakistan $(n=286)$

\begin{tabular}{|l|l|c|c|c|c|c|c|}
\hline Variable & Attributes & $\begin{array}{c}\text { Observed } \\
\text { counts }\end{array}$ & $\begin{array}{c}\text { Observed } \\
\text { \%ages }\end{array}$ & $\begin{array}{c}\text { Expected } \\
\text { counts }\end{array}$ & $\begin{array}{c}\text { Expected } \\
\text { \%ages }\end{array}$ & $\begin{array}{c}\text { Adjusted } \\
\text { expected } \\
\text { counts }\end{array}$ & $\begin{array}{c}\text { Adjusted ex- } \\
\text { pected \% }\end{array}$ \\
\hline Sex & Men & 123 & $\begin{array}{c}123^{*} 100 / 286 \\
=43 \%\end{array}$ & 664 & $\begin{array}{c}664^{*} 100 / 1250 \\
=53.12 \%\end{array}$ & $\begin{array}{c}664^{*} 286 / 1250 \\
=151.92\end{array}$ & $\begin{array}{c}151.92^{*} 100 / 286 \\
=53.12 \%\end{array}$ \\
\cline { 2 - 8 } & Women & 163 & $\begin{array}{c}163^{*} 100 / 286 \\
=57 \%\end{array}$ & 586 & $\begin{array}{c}586^{*} 100 / 1250 \\
=46.88 \%\end{array}$ & $\begin{array}{c}586^{\star} 286 / 1250 \\
=134.08\end{array}$ & $\begin{array}{c}134.08^{*} 100 / 286 \\
=46.88 \%\end{array}$ \\
\hline \multicolumn{2}{|c|}{ Total } & 286 & $100 \%$ & 1250 & $100.00 \%$ & 286.00 & $100.00 \%$ \\
\hline
\end{tabular}

Table 3.2.1.2: Comparison of observed (sample) to the expected (population) distribution of DR-TB population by sex in D.I.Khan Division, Pakistan $(n=286)$

\begin{tabular}{|l|l|c|c|c|c|c|c|c|c|}
\hline Variable & Attributes & $\mathrm{O}$ & $\mathrm{E}$ & $\mathrm{O}-\mathrm{E}$ & $(\mathrm{O}-\mathrm{E})^{2}$ & $(\mathrm{O}-\mathrm{E})^{2} / \mathrm{E}$ & $\chi^{2}$ & d.f. & p-value \\
\hline \multirow{3}{*}{ Sex } & Men & 123 & 151.92 & -28.92 & 836.37 & 5.51 & 11.743 & 1 & .00061 \\
\cline { 2 - 9 } & Women & 163 & 134.08 & 28.92 & 836.37 & 6.24 & $\mathrm{H}_{01}$ rejected at alpha .05 \\
\hline \multicolumn{2}{|c|}{ Total } & 286 & 286.00 & \multicolumn{5}{c|}{ Chi-square goodness of fit with Yates correction } \\
\hline
\end{tabular}

$\mathrm{O}=$ Observed count, $\mathrm{E}=$ Expected count, $\mathrm{X}^{2}=$ Chi-square statistics, d.f. $=$ degree of freedom

Table 3.2.2.1:Observed, expected and adjusted expected counts and percentages for distribution of DR-TB population by age groups in D.I.Khan Division, Pakistan $(n=286)$

\begin{tabular}{|l|l|c|c|c|c|c|c|}
\hline Variable & Attributes & $\begin{array}{c}\text { Observed } \\
\text { counts }\end{array}$ & $\begin{array}{c}\text { Observed } \\
\text { \%ages }\end{array}$ & $\begin{array}{c}\text { Expected } \\
\text { counts }\end{array}$ & $\begin{array}{c}\text { Expected } \\
\% \text { ages }\end{array}$ & $\begin{array}{c}\text { Adjusted ex- } \\
\text { pected counts }\end{array}$ & $\begin{array}{c}\text { Adjusted expect- } \\
\text { ed \% }\end{array}$ \\
\hline \multirow{3}{*}{$\begin{array}{l}\text { Age } \\
\text { groups }\end{array}$} & $\begin{array}{l}0-14 \\
\text { years }\end{array}$ & 8 & 2.80 & 31 & $\begin{array}{c}31^{*} 100 / 1250 \\
=2.48 \%\end{array}$ & $\begin{array}{c}31^{\star} 286 / 1250 \\
=7.09\end{array}$ & $\begin{array}{c}7.09 * 100 / 286 \\
=2.48 \%\end{array}$ \\
\cline { 2 - 8 } & $\begin{array}{l}15-44 \\
\text { years }\end{array}$ & 172 & 60.14 & 996 & $\begin{array}{c}996 * 100 / 1250 \\
=79.68 \%\end{array}$ & $\begin{array}{c}996 * 286 / 1250 \\
=227.89\end{array}$ & $\begin{array}{c}227.89 * 100 / 286 \\
=79.68 \%\end{array}$ \\
\cline { 2 - 8 } & $\begin{array}{l}\geq 45 \\
\text { years }\end{array}$ & 106 & 37.06 & 223 & $\begin{array}{c}223 * 100 / 1250 \\
=17.84 \%\end{array}$ & $\begin{array}{c}223^{\star} 286 / 1250 \\
=51.02\end{array}$ & $\begin{array}{c}51.02 * 100 / 286 \\
=17.84 \%\end{array}$ \\
\hline Total & 286 & $100.00 \%$ & & $100.00 \%$ & 286.00 & $100.00 \%$ \\
\hline
\end{tabular}


Distribution of DR-TB by sex, age groups, occupation, province, division, district, type of disease, type...

of-fit test, which showed p-value <alpha. $\mathrm{H}_{02}$ was declared as false and therefore rejected, showing that the observations did not fit the statistical model of the population. In simple words, our observed prevalence (distribution by age groups) of DR-TB in different age groups was not similar to expected prevalence by Akhter, et al. ${ }^{9}$ (Table 3.2.2.2)

3.2.3 Distribution of DR-TB by occupation (RQ 3 \& $\left.13, H_{03}\right)$ : The observed distribution of DR-TB by occupation in sample was compared to the assumed expected distribution in the population through chisquare goodness-of-fit test. The assumed expected distribution was estimated from Wahab, et al. ${ }^{10}$ which showed distribution of 30 MDR-TB cases as 11 (36.67 \%) labourer, 01 (3.33\%) student, 02 (6.67\%) govt. employee, 13 (43.33\%) housewife and $03(10 \%)$ others. $\mathrm{H}_{03}$ proved to be true, hence accepted, showing that the observed distribution of DR-TB by occupation in sample is similar to expected for population. (Tables 3.2.3.1 \& 3.2.3.2)

Table 3.2.2.2: Comparison of observed (sample) to the expected (population) distribution of DR-TB population by age groups in D.I.Khan Division, Pakistan $(n=286)$

\begin{tabular}{|l|l|c|c|c|c|c|c|c|c|}
\hline Variable & Attributes & $\mathrm{O}$ & $\mathrm{E}$ & $\mathrm{O}-\mathrm{E}$ & $(\mathrm{O}-\mathrm{E})^{2}$ & $(\mathrm{O}-\mathrm{E})^{2} / \mathrm{E}$ & $\mathrm{X}^{2}$ & d.f. & $\mathrm{p}$-value \\
\hline \multirow{3}{*}{ Age groups } & $0-14$ years & 8 & 7.09 & 0.91 & 0.83 & 0.12 & \multirow{3}{*}{73.071} & \multirow{3}{*}{2} & $<.00001$ \\
\cline { 2 - 10 } & $15-44$ years & 172 & 227.89 & -55.89 & 3123.69 & 13.71 & & & \multicolumn{2}{c|}{$\mathrm{H}_{02}$ rejected at alpha .05} \\
\cline { 2 - 10 } & $\geq 45$ years & 106 & 51.02 & 54.98 & 3022.80 & 59.25 & \multicolumn{7}{c|}{ Chi-square goodness of fit with Yates correction } \\
\hline
\end{tabular}

$\mathrm{O}=$ Observed count, $\mathrm{E}=$ Expected count, $\chi^{2}=$ Chi-square statistics, d.f. $=$ degree of freedom

Table 3.2.3.1: Observed and expected counts and percentages for distribution of DR-TB population by occupation in D.I.Khan Division, Pakistan $(n=286)$

\begin{tabular}{|l|l|c|c|c|c|}
\hline Variable & Attributes & $\begin{array}{c}\text { Observed } \\
\text { counts }\end{array}$ & Observed \%ages & $\begin{array}{c}\text { Expected } \\
\text { counts }\end{array}$ & Expected \%ages \\
\hline \multirow{3}{*}{ Occupation } & Office worker & 33 & $33 * 100 / 286=11.54 \%$ & 29 & $29 * 100 / 286=10.14 \%$ \\
\cline { 2 - 6 } & Labor & 113 & $113 * 100 / 286=39.51 \%$ & 115 & $115^{\star} 100 / 286=40.21 \%$ \\
\cline { 2 - 6 } & House wife & 140 & $140 * 100 / 286=48.95 \%$ & 142 & $142 * 100 / 286=49.65 \%$ \\
\hline \multicolumn{2}{|c|}{ Total } & 286 & $100.00 \%$ & 286 & $100.00 \%$ \\
\hline
\end{tabular}

Table 3.2.3.2: Comparison of observed (sample) to the expected (population) distribution of DR-TB population by occupation in D.I.Khan Division, Pakistan $(n=286)$

\begin{tabular}{|l|l|c|c|c|c|c|c|c|c|}
\hline S. No. & Occupation & $\mathrm{O}$ & $\mathrm{E}$ & $\mathrm{O}-\mathrm{E}$ & $(\mathrm{O}-\mathrm{E})^{2}$ & $(\mathrm{O}-\mathrm{E})^{2} / \mathrm{E}$ & $\mathrm{X}^{2}$ & $\mathrm{~d} . \mathrm{f}$ & $\mathrm{p}$-value \\
\hline 1 & Office worker & 33 & 29 & 4 & 16 & 0.55 & \multirow{2}{*}{0.615} & \multirow{2}{*}{2} & \multirow{2}{*}{.7354} \\
\hline 2 & Labor & 113 & 115 & -2 & 4 & 0.03 & & & \\
\hline 3 & House wife & 140 & 142 & -2 & 4 & 0.03 & $\mathrm{H}_{03}$ accepted at alpha 0.05 \\
\hline \multicolumn{7}{|l|}{ Total } & 286 & 286 & \multicolumn{7}{|c|}{ Chi-square goodness of fit with Yates correction } \\
\hline
\end{tabular}

$\mathrm{O}=$ Observed count, $\mathrm{E}=$ Expected count, $\chi^{2}=$ Chi-square value, d.f. $=$ degree of freedom

Table 3.2.4.1: Observed and expected counts and percentages for distribution of DR-TB population by province in D.I.Khan Division, Pakistan $(n=286)$

\begin{tabular}{|l|l|c|c|c|c|}
\hline S. No. & Provinces & $\begin{array}{c}\text { Observed } \\
\text { counts }\end{array}$ & Observed \%ages & $\begin{array}{c}\text { Expected } \\
\text { counts }\end{array}$ & Expected \%ages \\
\hline 1 & Khyber Pakhtunkhwa & 175 & $175^{*} 100 / 286=61.19 \%$ & 177 & $177^{*} 100 / 286=61.89 \%$ \\
\hline 2 & Punjab & 56 & $56 * 100 / 286=19.58 \%$ & 46 & $46 * 100 / 286=16.08 \%$ \\
\hline 3 & Baluchistan & 9 & $9 * 100 / 286=03.15 \%$ & 12 & $12 * 100 / 286=4.20 \%$ \\
\hline 4 & Sindh & 1 & $1 * 100 / 286=00.35 \%$ & 1 & $1 * 100 / 286=0.35 \%$ \\
\hline 5 & FATA & 45 & $45^{*} 100 / 286=15.73 \%$ & 50 & $50 * 100 / 286=17.48 \%$ \\
\hline & Total & 286 & $100.00 \%$ & 286 & $100.00 \%$ \\
\hline
\end{tabular}


3.2.4 Distribution of DR-TB by province (RQ 4 \& 14, $\mathrm{H}_{04}$ ): The observed distribution of DR-TB by province in sample was compared to the hypothetical expected distribution in the population through chi-square goodness-of-fit test. $\mathrm{H}_{04}$ proved to be true, hence accepted showing that the observed distribution of DR-TB by province in sample is similar to hypothetical expected distribution for population. (Tables 3.2.4.1 \& 3.2.4.2)

3.2.5 Distribution of DR-TB by Division (RQ 5 \& 15, $\mathbf{H}_{05}$ ): The observed distribution of DR-TB by Division in sample was compared to the hypothetical expected distribution in the population through chi-square goodness-of-fit test. $\mathrm{H}_{05}$ proved to be true, hence accepted showing that the observed distribution of DR-TB by division in sample is similar to hypothetical expected distribution for population. (Tables 3.2.5.1 \& 3.2.5.2)

3.2.6 Distribution of DR-TB by district (RQ $6 \& 16$, $\mathbf{H}_{06}$ ): The observed distribution of DR-TB by district in sample was compared to the hypothetical expected distribution in the population through chi-square goodness-of-fit test. $\mathrm{H}_{06}$ proved to be true, hence

Table 3.2.4.2: Comparison of observed (sample) to the expected (population) distribution of DR-TB population by province in D.I.Khan Division, Pakistan $(n=286)$

\begin{tabular}{|c|c|c|c|c|c|c|c|c|c|}
\hline S. No. & Provinces & $\mathrm{O}$ & E & O-E & $(O-E)^{2}$ & $(\mathrm{O}-\mathrm{E})^{2} / \mathrm{E}$ & $x^{2}$ & d.f & $\mathrm{p}$-value \\
\hline 1 & Khyber Pakhtunkhwa & 175 & 177 & -2 & 4 & 0.02 & 3.447 & 4 & .48606 \\
\hline 2 & Punjab & 56 & 46 & 10 & 100 & 2.17 & \multirow{4}{*}{\multicolumn{3}{|c|}{$\mathrm{H}_{04}$ accepted at alpha 0.05}} \\
\hline 3 & Baluchistan & 9 & 12 & -3 & 9 & 0.75 & & & \\
\hline 4 & Sindh & 1 & 1 & 0 & 0 & 0.00 & & & \\
\hline 5 & FATA & 45 & 50 & -5 & 25 & 0.50 & & & \\
\hline & Total & 286 & 286 & \multicolumn{6}{|c|}{ Chi-square goodness of fit with Yates correction } \\
\hline
\end{tabular}

Table 3.2.5.1: Observed and expected counts and percentages for distribution of DR-TB population by division in D.I.Khan Division, Pakistan $(n=286)$

\begin{tabular}{|l|l|c|c|c|c|}
\hline S. No. & Division & Observed counts & Observed \%ages & Expected counts & Expected \%ages \\
\hline 1 & D.I.Khan & 178 & $178 * 100 / 286=62.24 \%$ & 181 & $181 * 100 / 286=63.29 \%$ \\
\hline 2 & Bannu & 40 & $40 * 100 / 286=13.99 \%$ & 43 & $43 * 100 / 286=15.03 \%$ \\
\hline 3 & Sargodha & 56 & $56 * 100 / 286=19.58 \%$ & 50 & $50 * 100 / 286=17.48 \%$ \\
\hline 4 & Zhob & 9 & $9 * 100 / 286=3.15 \%$ & 11 & $11 * 100 / 286=3.85 \%$ \\
\hline 5 & Others & 3 & $3 * 100 / 286=1.04 \%$ & 1 & $1 * 100 / 286=0.35 \%$ \\
\hline \multicolumn{2}{r|}{ Total } & 286 & $100.00 \%$ & 286 & $100.00 \%$ \\
\hline
\end{tabular}

Table 3.2.5.2: Comparison of observed (sample) to the expected (population) distribution of DR-TB population by division in D.I.Khan Division, Pakistan $(n=286)$

\begin{tabular}{|c|c|c|c|c|c|c|c|c|c|}
\hline S. No. & Division & O & E & O-E & $(\mathrm{O}-\mathrm{E})^{2}$ & $(\mathrm{O}-\mathrm{E})^{2} / \mathrm{E}$ & $x^{2}$ & d.f & p-value \\
\hline 1 & D.I.Khan & 178 & 181 & -3 & 9 & 0.05 & \multirow{4}{*}{5.343} & \multirow{4}{*}{4} & \multirow{4}{*}{.25391} \\
\hline 2 & Bannu & 40 & 43 & -3 & 9 & 0.21 & & & \\
\hline 3 & Sargodha & 56 & 50 & 6 & 36 & 0.72 & & & \\
\hline 4 & Zhob & 9 & 11 & -2 & 4 & 0.36 & & & \\
\hline 5 & Others & 3 & 1 & 2 & 4 & 4.00 & \multicolumn{3}{|c|}{$\mathrm{H}_{05}$ accepted at alpha .05} \\
\hline & Total & 286 & 286 & \multicolumn{6}{|c|}{ Chi-square goodness of fit with Yates correction } \\
\hline
\end{tabular}

$\mathrm{O}=$ Observed count, $\mathrm{E}=$ Expected count, $\chi^{2}=$ Chi-square value, d.f. $=$ degree of freedom 
accepted showing that the observed distribution of DR-TB by district in sample is similar to hypothetical expected distribution for population. (Tables 3.2.6.1 \& 3.2.6.2)

\subsubsection{Distribution of DR-TB by type of disease} $\left(R Q 7 \&\right.$ \& $\left.7, H_{07}\right)$ : Our observed distribution for pulmonary versus extra-pulmonary TB was 282:4 from a sample of 286 against expected counts of 1231:19 from 1,250 previously treated DR-TB cases by Akhter, et al. ${ }^{9}$ These were not comparable due to different sample sizes/ denominators. Hence the expected counts and expected percentages were adjusted for a sample of 286 . The expected counts of 1231:19 were replaced by 281.65:4.35 (adjusted expected). Adjusted expected percentages came similar to expected percentages, so not changed. (Table 3.2.7.1)

The observed distribution of DR-TB by type of disease in sample was compared to the expected distribution in the population through chi-square goodness-of-fit test, which showed p-value >alpha. $\mathrm{H}_{07}$ was declared as true and therefore accepted, showing that the observations fit the statistical model of the population. In simple words, our observed prevalence of DR-TB for pulmonary TB $98.60 \%$ was statistically similar to what we expected (adjusted) for

Table 3.2.6.1: Observed and expected counts and percentages for distribution of DR-TB population by districts in D.I.Khan Division, Pakistan $(n=286)$

\begin{tabular}{|l|l|c|c|c|c|}
\hline S. No. & Districts & $\begin{array}{c}\text { Observed } \\
\text { counts }\end{array}$ & Observed \%ages & $\begin{array}{c}\text { Expected } \\
\text { counts }\end{array}$ & Expected \%ages \\
\hline 1 & D.I.Khan & 121 & $121 * 100 / 286=42.31 \%$ & 125 & $125 * 100 / 286=43.71 \%$ \\
\hline 2 & Tank & 18 & $18^{*} 100 / 286=06.29 \%$ & 22 & $22 * 100 / 286=7.69 \%$ \\
\hline 3 & Lakki Marwat & 27 & $27 * 100 / 286=09.44 \%$ & 19 & $19 * 100 / 286=6.64 \%$ \\
\hline 4 & Bannu & 9 & $9 * 100 / 286=03.15 \%$ & 8 & $8 * 100 / 286=2.80 \%$ \\
\hline 5 & Bhakkar & 49 & $49 * 100 / 286=17.13 \%$ & 50 & $50 * 100 / 286=17.48 \%$ \\
\hline 6 & Mianwali & 5 & $5 * 100 / 286=01.75 \%$ & 4 & $4 * 100 / 286=1.40 \%$ \\
\hline 7 & Zhob & 9 & $9 * 100 / 286=03.15 \%$ & 10 & $10 * 100 / 286=3.50 \%$ \\
\hline 8 & South Waziristan Agency & 41 & $41^{*} 100 / 286=14.33 \%$ & 42 & $42 * 100 / 286=14.68 \%$ \\
\hline 9 & North Waziristan Agency & 4 & $4 * 100 / 286=1.40 \%$ & 5 & $5 * 100 / 286=1.75 \%$ \\
\hline 10 & Others & 3 & $3 * 100 / 286=1.05 \%$ & 1 & $1 * 100 / 286=0.35 \%$ \\
\hline \multicolumn{2}{|c|}{ Total } & 286 & $100.00 \%$ & 286 & $100.00 \%$ \\
\hline
\end{tabular}

Table 3.2.6.2: Comparison of observed (sample) to the expected (population) distribution of DR-TB population by district in D.I.Khan Division, Pakistan $(\mathbf{n}=\mathbf{2 8 6})$

\begin{tabular}{|c|c|c|c|c|c|c|c|c|c|}
\hline S. No. & Districts & $\mathrm{O}$ & $\mathrm{E}$ & O-E & $(O-E)^{2}$ & $(O-E)^{2} / E$ & $x^{2}$ & d.f & $p$-value \\
\hline 1 & D.I.Khan & 121 & 125 & -4 & 16 & 0.13 & \multirow{9}{*}{8.943} & \multirow{9}{*}{9} & \multirow{9}{*}{.4426} \\
\hline 2 & Tank & 18 & 22 & -4 & 16 & 0.73 & & & \\
\hline 3 & Lakki Marwat & 27 & 19 & 8 & 64 & 3.37 & & & \\
\hline 4 & Bannu & 9 & 8 & 1 & 1 & 0.13 & & & \\
\hline 5 & Bhakkar & 49 & 50 & -1 & 1 & 0.02 & & & \\
\hline 6 & Mianwali & 5 & 4 & 1 & 1 & 0.25 & & & \\
\hline 7 & Zhob & 9 & 10 & -1 & 1 & 0.10 & & & \\
\hline 8 & South Waziristan Agency & 41 & 42 & -1 & 1 & 0.02 & & & \\
\hline 9 & North Waziristan Agency & 4 & 5 & -1 & 1 & 0.20 & & & \\
\hline 10 & Others & 3 & 1 & 2 & 4 & 4.00 & \multicolumn{3}{|c|}{$\mathrm{H}_{06}$ accepted at alpha .05} \\
\hline & Total & 286 & 286 & \multicolumn{6}{|c|}{ Chi-square goodness of fit with Yates correction } \\
\hline
\end{tabular}

$\mathrm{O}=$ Observed count, $\mathrm{E}=$ Expected count, $\mathrm{X}^{2}=$ Chi-square value, d.f. $=$ degree of freedom 
pulmonary TB $98.48 \%$ \& our observed prevalence of DR-TB for extra-pulmonary TB $1.40 \%$ was also similar to what we expected (adjusted) for extra-pulmonary TB 1.52\% from Akhter, et al ${ }^{9}$. (Table 3.2.7.2)

3.2.8 Distribution of DR-TB by type of drug resistance $\left(R Q \quad 8\right.$ \& 18, $\left.H_{08}\right)$ : Our observed distribution for mono-resistant: poly-resistant: MDR: XDR-TB was 1:2:273:10 from a sample of 286 against expected counts of 10:6:260:13 from 289 DR-TB cases by Ahmad, et al. ${ }^{11}$. These were not comparable due to different sample sizes/ denominators. Hence the expected counts and expected percentages were adjusted for a sample of 286. The expected counts of 10:6:260:13 were replaced by $9.90: 5.94: 257.30: 12.86$ (adjusted expected). Adjusted expected percentages came similar to expected percentages, so not changed. (Table 3.2.8.1)

The observed distribution of DR-TB by type of drug resistance in sample was compared to the expected distribution in the population through chi-square goodness-of-fit test, which showed p-value < alpha. $\mathrm{H}_{08}$ was declared as false and therefore rejected, showing that the observations not fit the statistical model of the population. In simple words, our ob-

Table 3.2.7.1: Observed, expected and adjusted expected counts and percentages for distribution of DR-TB population by type of disease in D.I.Khan Division, Pakistan $(n=286)$

\begin{tabular}{|c|l|c|c|c|c|c|c|}
\hline Variable & Attributes & $\begin{array}{c}\text { Observed } \\
\text { counts }\end{array}$ & $\begin{array}{c}\text { Observed } \\
\text { \%ages }\end{array}$ & $\begin{array}{c}\text { Expected } \\
\text { counts }\end{array}$ & $\begin{array}{c}\text { Expected } \\
\% \text { ages }\end{array}$ & $\begin{array}{c}\text { Adjusted ex- } \\
\text { pected counts }\end{array}$ & $\begin{array}{c}\text { Adjusted ex- } \\
\text { pected \% }\end{array}$ \\
\hline \multirow{2}{*}{$\begin{array}{l}\text { Type of } \\
\text { disease }\end{array}$} & $\begin{array}{l}\text { Pulmonary } \\
\text { TB }\end{array}$ & 282 & $\begin{array}{c}282^{*} 100 / 286 \\
=98.60 \%\end{array}$ & 1231 & $\begin{array}{c}1231 * 100 / 1250 \\
=98.48 \%\end{array}$ & $\begin{array}{c}1231 * 286 / 1250 \\
=281.65\end{array}$ & $\begin{array}{c}281.65 * 100 / 286 \\
=98.48 \%\end{array}$ \\
\cline { 2 - 8 } & $\begin{array}{l}\text { Extra pul- } \\
\text { monary TB }\end{array}$ & 4 & $\begin{array}{c}4^{*} 100 / 286 \\
=1.40 \%\end{array}$ & 19 & $\begin{array}{c}19 * 100 / 1250 \\
=1.52 \%\end{array}$ & $\begin{array}{c}19 * 286 / 1250 \\
=4.35\end{array}$ & $\begin{array}{c}4.35 * 100 / 286 \\
=1.52 \%\end{array}$ \\
\hline \multicolumn{2}{|c|}{ Total } & 286 & $100.00 \%$ & 1250 & $100.00 \%$ & 286.00 & $100.00 \%$ \\
\hline
\end{tabular}

Table 3.2.7.2: Comparison of observed (sample) to the expected (population) distribution of DR-TB population by type of disease in D.I.Khan Division, Pakistan $(n=286)$

\begin{tabular}{|l|l|c|c|c|c|c|c|c|c|}
\hline Variable & Attributes & $\mathrm{O}$ & $\mathrm{E}$ & $\mathrm{O}-\mathrm{E}$ & $(\mathrm{O}-\mathrm{E})^{2}$ & $(\mathrm{O}-\mathrm{E})^{2} / \mathrm{E}$ & $\chi^{2}$ & d.f & $\mathrm{p}$-value \\
\hline \multirow{2}{*}{$\begin{array}{l}\text { Type of } \\
\text { disease }\end{array}$} & Pulmonary & 282 & 281.65 & 0.35 & 0.1225 & 0.00 & 0.028 & 1 & .86572 \\
\cline { 2 - 10 } & Extra-pulmonary & 4 & 4.35 & -0.35 & 0.1225 & 0.028 & $\mathrm{H}_{07}$ accepted at alpha .05 \\
\hline \multicolumn{3}{|c|}{ Total } & 286 & 286.00 & \multicolumn{7}{c|}{ Chi-square goodness of fit with Yates correction } \\
\hline
\end{tabular}

$\mathrm{O}=$ Observed count, $\mathrm{E}=$ Expected count, $\mathrm{X}^{2}=$ Chi-square value, d.f. $=$ degree of freedom

Table 3.2.8.1: Observed, expected and adjusted expected counts and percentages for distribution of DR-TB population by drug resistance in D.I.Khan Division, Pakistan $(n=286)$

\begin{tabular}{|c|c|c|c|c|c|c|c|}
\hline Variable & Attributes & $\begin{array}{c}\text { Observed } \\
\text { counts }\end{array}$ & $\begin{array}{c}\text { Observed } \\
\text { \%ages }\end{array}$ & $\begin{array}{l}\text { Expected } \\
\text { counts }\end{array}$ & $\begin{array}{l}\text { Expected } \\
\text { \%ages }\end{array}$ & $\begin{array}{l}\text { Adjusted ex- } \\
\text { pected counts }\end{array}$ & $\begin{array}{l}\text { Adjusted ex- } \\
\text { pected \% }\end{array}$ \\
\hline \multirow{4}{*}{$\begin{array}{l}\text { Type of } \\
\text { drug re- } \\
\text { sistance }\end{array}$} & $\begin{array}{l}\text { Mono-re- } \\
\text { sistant }\end{array}$ & 1 & $\begin{array}{c}1 * 100 / 286 \\
=0.35 \%\end{array}$ & 10 & $\begin{array}{c}10 * 100 / 289 \\
=3.46 \%\end{array}$ & $\begin{array}{c}10 * 286 / 289 \\
=9.90\end{array}$ & $\begin{array}{c}9.90 * 100 / 286 \\
=3.46 \%\end{array}$ \\
\hline & $\begin{array}{l}\text { Poly-re- } \\
\text { sistant }\end{array}$ & 2 & $\begin{array}{c}2 * 100 / 286 \\
=0.70 \%\end{array}$ & 6 & $\begin{array}{c}6 * 100 / 289 \\
=2.08 \%\end{array}$ & $\begin{array}{c}6 * 286 / 289 \\
=5.94\end{array}$ & $\begin{array}{c}5.94 * 100 / 286 \\
=2.08 \%\end{array}$ \\
\hline & MDR & 273 & $\begin{array}{c}273 * 100 / 286 \\
=95.45 \%\end{array}$ & 260 & $\begin{array}{c}260 * 100 / 289 \\
=89.96 \%\end{array}$ & $\begin{array}{c}260 * 286 / 289 \\
=257.30\end{array}$ & $\begin{array}{c}257.30 * 100 / 286 \\
\quad=89.96 \%\end{array}$ \\
\hline & XDR & 10 & $\begin{array}{c}10 * 100 / 286 \\
=3.50 \%\end{array}$ & 13 & $\begin{array}{c}13 * 100 / 289 \\
=4.50 \%\end{array}$ & $\begin{array}{c}13 * 286 / 289= \\
12.86\end{array}$ & $\begin{array}{c}12.86 * 100 / 286 \\
=4.50 \%\end{array}$ \\
\hline \multicolumn{2}{|c|}{ Total } & 286 & $100.00 \%$ & 289 & $100.00 \%$ & 286.00 & $100.00 \%$ \\
\hline
\end{tabular}


served prevalence of DR-TB in different attributes of drug resistance was not matching to the expected prevalence for population by Ahmad, et al. ${ }^{11}$ (Table 3.2.8.2)

3.2.9 Distribution of MDR-TB by treatment regimen (RQ 9 \& 19, $\left.H_{09}\right)$ : Our observed distribution for STR versus LTR was 27:246 from a sample of 273 MDR-TB cases against expected counts of 67:65 from 132 MDR-TB cases by Arshad, et al. ${ }^{12}$ These were not comparable due to different sample sizes/ denominators. Hence the expected counts and expected percentages were adjusted for a sample of 273. The expected counts of 67:65 were replaced by 138.57:134.43 (adjusted expected). Adjusted expected percentages came similar to expected percentages, so not changed. (Table 3.2.9.1)

The observed distribution of DR-TB by treatment regimen in sample was compared to the expected distribution in the population through chi-square goodness-of-fit test, which showed p-value < alpha. $\mathrm{H}_{09}$ was declared as false and therefore rejected, showing that the observations did not fit the statistical model of the population. In simple words, our observed prevalence of MDR-TB patients on STR $9.89 \% \%$ was significantly lower to what we expected (adjusted) for MDR-TB patients on STR $50.76 \%$ \& our observed prevalence of MDR-TB patients on LTR $90.11 \%$ was higher to what we expected (adjusted) for MDR-TB patients on LTR $49.24 \%$ as compared to the prevalence by Arshad, et al. ${ }^{12}$ (Table 3.2.9.2)

3.2.10 Distribution of DR-TB by type of outcome of treatment $\left(R Q 10 \& 20, H_{10}\right)$ : The observed distribution of DR-TB by outcome of treatment in sample was compared to the expected distribution in the population through chi-square goodness-of-fit test. The expected counts for outcome were estimated from a systematic review and meta-analysis by Johnston, et al. ${ }^{4}$ from Canada and Alene, et al. ${ }^{6}$

Table 3.2.8.2: Comparison of observed (sample) to the expected (population) distribution of DR-TB population by type of drug resistance in D.I.Khan Division, Pakistan $(n=286)$

\begin{tabular}{|c|c|c|c|c|c|c|c|c|c|}
\hline S. No. & $\begin{array}{l}\text { Type of drug } \\
\text { resistance }\end{array}$ & $\mathrm{O}$ & $E$ & O-E & $(O-E)^{2}$ & $(O-E)^{2} / E$ & $x^{2}$ & d.f & $\mathrm{p}$-value \\
\hline 1 & Mono-resistant & 1 & 9.90 & -8.90 & 79.21 & 8.00 & \multirow{3}{*}{12.208} & \multirow{3}{*}{3} & \multirow{3}{*}{.0067} \\
\hline 2 & Poly-resistant & 2 & 5.94 & -3.94 & 15.52 & 2.61 & & & \\
\hline 3 & MDR & 273 & 257.30 & 15.70 & 246.49 & 0.96 & & & \\
\hline 4 & XDR & 10 & 12.86 & -2.86 & 8.18 & 0.64 & \multicolumn{3}{|c|}{$\mathrm{H}_{08}$ rejected at alpha 0.05} \\
\hline & Total & 286 & 286.00 & \multicolumn{6}{|c|}{ Chi-square goodness of fit with Yates correction } \\
\hline
\end{tabular}

Table 3.2.9.1: Observed, expected and adjusted expected counts and percentages for distribution of MDR-TB population by type of regimen in D.I.Khan Division, Pakistan $(n=273)$

\begin{tabular}{|c|c|c|c|c|c|c|c|}
\hline Variable & Attributes & $\begin{array}{c}\text { Observed } \\
\text { counts }\end{array}$ & $\begin{array}{c}\text { Observed } \\
\text { \%ages }\end{array}$ & $\begin{array}{c}\text { Expected } \\
\text { counts }\end{array}$ & $\begin{array}{c}\text { Expected } \\
\text { \%ages }\end{array}$ & $\begin{array}{c}\text { Adjusted ex- } \\
\text { pected counts }\end{array}$ & $\begin{array}{c}\text { Adjusted expected } \\
\% \text { ages }\end{array}$ \\
\hline \multirow{3}{*}{ Sex } & STR & 27 & $\begin{array}{c}27^{*} 100 / 273 \\
=9.89 \%\end{array}$ & 67 & $\begin{array}{c}67^{*} 100 / 132 \\
=50.76 \%\end{array}$ & $\begin{array}{c}67^{\star} 273 / 132 \\
=138.57\end{array}$ & $\begin{array}{c}138.57^{*} 100 / 273 \\
=50.76 \%\end{array}$ \\
\cline { 2 - 8 } & LTR & 246 & $\begin{array}{c}246 * 100 / 273 \\
=90.11 \%\end{array}$ & 65 & $\begin{array}{c}65^{*} 100 / 132 \\
=49.24 \%\end{array}$ & $\begin{array}{c}65^{\star} 273 / 132 \\
=134.43\end{array}$ & $\begin{array}{c}134.43^{*} 100 / 273 \\
=49.24 \%\end{array}$ \\
\hline \multicolumn{2}{|c|}{ Total } & 273 & $100 \%$ & 132 & $100.00 \%$ & 273.00 & $100.00 \%$ \\
\hline
\end{tabular}

Table 3.2.9.2: Comparison of observed (sample) to the expected (population) distribution of MDR-TB population by treatment regimen in D.I.Khan Division, Pakistan $(n=273)$

\begin{tabular}{|l|l|c|c|c|c|c|c|c|c|}
\hline Variable & Attributes & $\mathrm{O}$ & $\mathrm{E}$ & $\mathrm{O}-\mathrm{E}$ & $(\mathrm{O}-\mathrm{E})^{2}$ & $(\mathrm{O}-\mathrm{E})^{2} / \mathrm{E}$ & $\mathrm{X}^{2}$ & d.f & $\mathrm{p}$-value \\
\hline \multirow{2}{*}{$\begin{array}{l}\text { Treatment } \\
\text { Regimen }\end{array}$} & STR & 27 & 138.57 & -111.57 & 12447.86 & 89.83 & 182.428 & 2 & $<.00001$ \\
\cline { 2 - 9 } & LTR & 246 & 134.43 & 111.57 & 12447.86 & 92.60 & $\mathrm{H}_{09}$ rejected at alpha 0.05 \\
\hline \multicolumn{2}{|c|}{ Total } & 273 & 273.00 & \multicolumn{3}{|c|}{ Chi-square goodness of fit with Yates correction } \\
\hline
\end{tabular}

$\mathrm{O}=$ Observed count, $\mathrm{E}=$ Expected count, $\chi^{2}=$ Chi-square value, d.f. $=$ degree of freedom 
from Changsha from Hunan Province of China. $\mathrm{H}_{010}$ proved to be false, hence rejected showing that the observed prevalence of DR-TB by outcome of treatment in sample was different from the expected prevalence obtained from the above two studies. ${ }^{4,6}$ (Tables 3.2.10.1 \& 3.2.10.2)

\section{DISCUSSION}

4.1 Distribution of DR-TB by sex (RQ $\left.1 \& 11, H_{01}\right)$ : In our study $(\mathrm{n}=286)$ prevalence of DR-TB was lower in men $43 \%(95 \% \mathrm{Cl} 37.39-48.80)$ than women $57 \%$ (95\% Cl 51.19-62.60). In contrast to our study, the following five studies showed higher prevalence in men than women. ${ }^{3,6,7,9,10}$ Akhter, et al. ${ }^{9}$ from Lahore, Pakistan distributed 1,250 retreatment DR-TB cases across men and women as $53.12 \%$ and $46.88 \%$ respectively. Wahab, et al. ${ }^{10}$ from Peshawar, Pakistan showed distribution of 30 MDR-TB cases as $56.7 \%$ men and $43.3 \%$ women. Alene, et al. ${ }^{6}$ from Changsha, China reported 480 DR-TB cases with $70.7 \%$ men and
29.3\% women. Venkatesh, et al. ${ }^{7}$ from Gorakhpur, India reported distribution of 157 MDR-TB patients as $68.8 \%(95 \% \mathrm{Cl} 61.1-75.5)$ in men and $31.2 \%(95 \% \mathrm{Cl}$ 24.4-38.8) in women. Worku, et al. ${ }^{3}$ from Addis Ababa, Ethiopia with 340 pulmonary MDR/RR-TB cases notified distribution as $52.94 \%$ men and $47.06 \%$ women.

No study similar to ours could be obtained from literature. Similarly no studies showing similar prevalence of DR-TB cases in men and women could be obtained.

Our observed prevalence in men $43 \%$ was lower to what we expected (adjusted) for men 53.12\% \& in women $57 \%$ it was higher to what we expected (adjusted) for women $46.88 \%$ from Akhter, et $\mathrm{al}^{9}$. (Table 3.2.1.2)

4.2 Distribution of DR-TB by age groups (RQ 2 \&12, $\mathrm{H}_{02}$ ):

In our study DR-TB was most prevalent in age group 15-44 years 60.14\%, (95\% Cl 54.37-65.81) (Table 3.1.2). Almost similar results were reported by Wa-

Table 3.2.10.1: Observed and expected counts and percentages for distribution of DR-TB population by outcome of treatment in D.I.Khan Division, Pakistan $(n=286)$

\begin{tabular}{|c|c|c|c|c|c|}
\hline S. No. & Outcome of Treatment & $\begin{array}{c}\text { Observed } \\
\text { counts }\end{array}$ & Observed \%ages & $\begin{array}{l}\text { Expected } \\
\text { counts }\end{array}$ & Expected \%ages \\
\hline 1 & Cured & 158 & $158 * 100 / 286=55.24 \%$ & 156 & $156 * 100 / 286=54.54 \%$ \\
\hline 2 & Treatment completed & 3 & $3 * 100 / 286=1.05 \%$ & 8 & $8 * 100 / 286=2.80 \%$ \\
\hline 3 & Died & 59 & $59 * 100 / 286=20.63 \%$ & 8 & $8 * 100 / 286=2.80 \%$ \\
\hline 4 & Failed & 9 & $9 * 100 / 286=3.15 \%$ & 38 & $38 * 100 / 286=13.29 \%$ \\
\hline 5 & Lost to follow up & 31 & $31 * 100 / 286=10.84 \%$ & 50 & $50 * 100 / 286=17.48 \%$ \\
\hline 6 & Not evaluated & 6 & $6 * 100 / 286=2.09 \%$ & 6 & $6 * 100 / 286=2.09 \%$ \\
\hline 7 & Still under treatment & 20 & $20 * 100 / 286=7.00 \%$ & 20 & $20 * 100 / 286=7.00 \%$ \\
\hline \multicolumn{2}{|r|}{ Total } & 286 & $100.00 \%$ & 286 & $100.00 \%$ \\
\hline
\end{tabular}

Table 3.2.10.2: Comparison of observed (sample) to the expected (population) distribution of DR-TB population by outcome of treatment in D.I.Khan Division, Pakistan $(n=286)$

\begin{tabular}{|c|c|c|c|c|c|c|c|c|c|}
\hline S. No. & $\begin{array}{l}\text { Outcome of } \\
\text { Treatment }\end{array}$ & $\mathrm{O}$ & $E$ & O-E & $(O-E)^{2}$ & $(\mathrm{O}-\mathrm{E})^{2} / \mathrm{E}$ & $x^{2}$ & d.f & p-value \\
\hline 1 & Cured & 158 & 156 & 2 & 4 & 0.03 & \multirow{6}{*}{357.627} & \multirow{6}{*}{6} & \multirow{6}{*}{$<.00001$} \\
\hline 2 & Treatment completed & 3 & 8 & -5 & 25 & 3.13 & & & \\
\hline 3 & Died & 59 & 8 & 51 & 2601 & 325.13 & & & \\
\hline 4 & Failed & 9 & 38 & -29 & 841 & 22.13 & & & \\
\hline 5 & Lost to follow up & 31 & 50 & -19 & 361 & 7.22 & & & \\
\hline 6 & Not evaluated & 6 & 6 & 0 & 0 & 0.00 & & & \\
\hline 7 & Still under treatment & 20 & 20 & 0 & 0 & 0.00 & \multicolumn{3}{|c|}{$\mathrm{H}_{10}$ rejected at alpha .05} \\
\hline & Total & 286 & 286 & \multicolumn{6}{|c|}{ Chi-square goodness of fit with Yates correction } \\
\hline
\end{tabular}

$\mathrm{O}=$ Observed count, $\mathrm{E}=$ Expected count, $\chi^{2}=$ Chi-square value, d.f. $=$ degree of freedom 
hab, et al. $^{10}(n=30$ MDR cases) $60 \%$ in $20-40$ years, Akhter, et al. ${ }^{9}(n=1,250$ retreatment DR-TB cases) $79.68 \%$ in $15-45$ years, Venkatesh, et al. ${ }^{7}(n=157$ MDR-TB cases) $79.7 \%$ in $18-40$ years and Worku, et al. ${ }^{3}$ ( $n=340$ pulmonary MDR/RR-TB cases) $89.4 \%$ in 15-44 years age group.

Our observed prevalence (distribution by age groups) in different age groups was different from expected prevalence by Akhter, et al. ${ }^{9}$ (Table 3.2.2.2)

4.3 Distribution of DR-TB by occupation (RQ 3 \& $13, \mathrm{H}_{03}$ ): Our study showed highest prevalence in housewife $48.95 \%$, followed by labourer $39.51 \%$ and office worker $11.54 \%$ (Table 3.1.2). Almost similar results were by Wahab, et al..$^{10}$ from Peshawar. On contrary Alene, et al. ${ }^{6}$ from Changsha, China reported highest prevalence in farmers $79.0 \%$.

Our observed distribution by occupation was similar to assumed expected distribution estimated from Wahab, et al. ${ }^{10}$ (Table 3.2.3.2)

4.4 Distribution of DR-TB by province (RQ 4 \& $\left.14, \mathrm{H}_{04}\right)$ : In our study Khyber Pakhtunkhwa province had highest number of DR-TB patients $61.19 \%$ (95\% Cl 55.42-66.65) (Table 3.1.4). No study could be retrieved for comparison. Our observed prevalence across different provinces of Pakistan in sample was similar to hypothetical expected distribution for population. (Table 3.2.4.2)

4.5 Distribution of DR-TB by Division (RQ 5 \& $15, \mathrm{H}_{05}$ ): In our study D.I.Khan Division had highest number of DR-TB patients $62.24 \%$ (95\% Cl 56.49 -67.66) (Table 3.1.5). No study could be retrieved from literature for comparison. Our observed prevalence across different divisions of Pakistan in sample was similar to hypothetical expected distribution for population. (Table 3.2.5.2)

4.6 Distribution of DR-TB by district (RQ 6 \& 16, $\mathrm{H}_{06}$ ): In our study D.I.Khan district had highest number of DR-TB patients $42.31 \%(95 \% \mathrm{Cl} 36.70$ 48.10) (Table 3.1.6). No study could be retrieved for comparison. Our observed prevalence across different districts of Pakistan in sample was similar to hypothetical expected distribution for population. (Table 3.2.6.2)

4.7 Distribution of DR-TB by type of disease (RQ 7 $\left.\& 17, \mathrm{H}_{07}\right)$ : Our study reported higher prevalence of DR-TB in pulmonary $98.60 \%(95 \% \mathrm{Cl} 96.45-99.45 \%)$ than extra-pulmonary cases $1.40 \%(95 \% \mathrm{Cl} 0.54-$ 3.54\%). Similar results were reported by Akhter, et al. ${ }^{9}$,Venkatesh, et al. ${ }^{7}$ and Ahmad, et al. ${ }^{11}$

Our observed prevalence for pulmonary TB $98.60 \%$ was similar to what we expected (adjusted) for pulmonary TB $98.48 \%$ \& our observed prevalence of extra-pulmonary TB $1.40 \%$ was also similar to what we expected (adjusted) for extra-pulmonary TB 1.52\% from Akhter, et al. ${ }^{9}$ (Table 3.2.7.2)

4.8 Distribution of DR-TB by type of drug resis- tance $\left(\mathrm{RQ} 8\right.$ \& 18, $\left.\mathrm{H}_{08}\right)$ :

In our study prevalence of MDR-TB was highest 273 (95.45\%) (Table 3.1.8). Ahmad, et al. ${ }^{11}$ from Peshawar reported 260 (89.96\%) total MDR-TB out of 289 DR-TB cases. Alene, et al. ${ }^{6}$ from Changsha, China reported 481 DR-TB cases of which 471 (98\%) were MDR-TB. $\mathrm{He}$, et al. ${ }^{5}$ from Shandong Province, China reported $29.35 \%(838 * 100 / 2,855=29.35 \%)$ MDR-TB out of 2,855 DR-TB cases.

Our observed prevalence of DR-TB in different attributes of drug resistance did not match to the expected prevalence for population by Ahmad, et al. ${ }^{11}$ (Table 3.2.8.2)

4.9 Distribution of MDR-TB by treatment regimen (RQ 9 \& 19, $H_{09}$ ): In our study out of 273 MDR-TB patients, $9.89 \%$ received STR while $90.11 \%$ LTR. Contrary to ours was study by Arshad, et al. ${ }^{12}$ from Peshawar who reported higher prevalence of 132 MDR-TB patients on STR $50.76 \%$ and lower prevalence of MDR-TB patients on LTR 49.24\%.

In simple words, our observed prevalence of MDR-TB patients on STR $9.89 \%$ was lower to what we expected (adjusted) for MDR-TB patients on STR $50.76 \%$ \& our observed prevalence of MDR-TB patients on LTR $90.11 \%$ was higher to what we expected (adjusted) for MDR-TB patients on LTR $49.24 \%$ by Arshad, et al. ${ }^{12}$ (Table 3.2.9.2)

4.10 Distribution of DR-TB by outcome of treatment (RQ 10 \& 20, $\left.\mathrm{H}_{20}\right)$ : Our study showed $56.29 \%(95 \% \mathrm{Cl} 50.50-61.92)$ completed treatment successfully (cured+ completed), $20.63 \%(95 \% \mathrm{Cl}$ $16.34-25.69)$ died, $3.15 \%(95 \% \mathrm{Cl} 1.66-5.87)$ failed treatment, $10.84 \%(95 \% \mathrm{Cl} 7.74-14.97)$ loss to follow up/defaulted, $2.09 \%(95 \% \mathrm{Cl} 0.96-4,50)$ not evaluated and $7 \%(95 \% \mathrm{Cl} 4.57-10.55)$ still under treatment (Table 3.1.10). Johnston, et al. ${ }^{4}$ from Canada $(n=4,959$ MDR-TB patients) reported $62 \%(95 \% \mathrm{Cl} 57-67)$ successful treatment, $11 \%(95 \% \mathrm{Cl} 9-13)$ died and $8 \%$ $(95 \% \mathrm{Cl} 5-11)$ failed therapy, default rate $13 \%(95 \% \mathrm{Cl}$ $9-17)$ and not evaluated $2 \%(95 \% \mathrm{Cl} 1-4)$. Alene, et al. ${ }^{6}$ from Changsha, China reported $(n=481$ DR-TB) $57.2 \%$ completed treatment successfully (cured +completed), $2.7 \%$ died, $13.1 \%$ failure and $27.0 \%$ loss to follow up. The proportion of successful treatment and not evaluated was similar in ours and the above mentioned studies. The proportion of death was higher while treatment failure and loss to follow up were lower in our sample than the above mentioned studies. $7 \%$ patients were still under treatment in our study, while other studies did not mention it. The observed distribution of DR-TB by outcome of treatment in sample was different from the expected distribution, estimated from the above two studies. ${ }^{4,6}$ (Table 3.2.10.2)

4.11 Innovative strengths of our study: Research is problem solving process for a defined population through a sample. We have drawn sample from our 
population at risk. Data of this sample was analyzed (descriptive statistics) and the population parameters were inferred from the analyzed data (estimation of parameter; inferential statistics). In this way population characteristics were inferred from sample, the ultimate purpose of research. Most global literature has no mention of their populations. Finally observed data of the sample was compared to the expected data from the population to see whether this sample is similar/ dissimilar to the population (hypothesis testing-inferential statistics).

We have arranged our research/study as per design of "Marwat's Logical Trajectory for Research Process", ${ }^{20-27}$ giving this effort a rational flow and including to sort out our research problems, knowledge gaps, converting research problems into research questions and research objectives, making research hypotheses and doing collection, analysis and interpretation of relevant data.

\section{CONCLUSIONS}

The prevalence of DR-TB was higher in women, age group 15-44 years, housewife, Khyber Pakhtunkhwa and D.I.Khan Division and District. Most common type of disease, drug resistance and treatment regimen was pulmonary TB, MDR and longer treatment respectively. Treatment success rate was $56.29 \%$. The observed prevalence by occupation, province, division, district and type of disease in sample was similar to population, while it was different by sex, age groups, type of drug resistance, regimen and treatment outcome.

Acknowledgement: We are thankful to Dr. Muhammad Marwat (marwatmuhammad@gmail.com) from Gomal Medical College, D.I.Khan for his overall supervision, his guidance and help in each step of the project from concept \& design, defining population \& drawing sample, data collection, analysis \& interpretation, writing this manuscript and allowing us to use his "Marwat's Logical Trajectory of Research Process".

\section{REFRENCES}

1. WHO. Global tuberculosis report 2019. World Health Organization. Geneva [accessed 2020 Dec 8]. Available at: https://www.who.int/tb/publications/global_report/en/

2. National TB Control Program. National guidelines for control of tuberculosis in Pakistan [revised and updated 2019, cited 2020 Apr 24]. Available at: http://ntp.gov.pk/ntp-old/uploads/National_Guidelines_for_TB_Revised_2019

3. Worku Y, Getinet T, Mohammed S, Yang Z. Drug-resistant tuberculosis in Ethiopia: characteristics of cases in a referral hospital and the implications. Int J Microbacteriol [serial online] 2018 [cited 2020 May 11]; 7:167-72. https://doi. org/10.4103/ijmy.ijmy_48_18
4. He XC, Zhang XX, Zhao JN, Liu Y, Yu CB, Yang $\mathrm{GR}$, et al. Epidemiological trends of drug-resistant tuberculosis in China from 2007 to 2014: a retrospective study. Medicine (Baltimore) [serial online] 2016 Apr [cited 2020 May 11]; 95(15): e3336. https://doi.org/10.1097/MD.0000000000003336

5. Johnston JC, Shahidi NC, Sadatsafavi M, Fitzgerald JM. Treatment outcomes of multidrug-resistant tuberculosis: a systematic review and meta-analysis. PLoS ONE [serial online] 2009 [cited 2020 May 11]; 4(9): e6914. https://doi. org/10.1371/journal.pone.0006914

6. Venkatesh U, Srivastava DK, Srivastava AK, Tiwari HC. Epidemiological profile of multidrug-resistant tuberculosis patients in Gorakhpur Division, Uttar Pradesh, India. J Family Med Prim Care [serial online] 2018 May-Jun [cited 2020 May 11]; 7(3): 589-95. https://doi.org/10.4103/jfmpc. jfmpc_99_17

7. Hasan R, Jabeen K, Ali A, Rafiq Y, Laiq R, Malik $B$, et al. Extensively drug-resistant tuberculosis in Pakistan. Emerg Infect Dis [serial online] 2010 Sep [cited 2020 May 11]; 16(9): 1473-5. https:// doi.org/10.3201/eid1609.100280

8. Ahmad N, Javed A, Sulaiman SAS, Ming LC, Ahmad I, Khan AH. Resistance patterns, prevalence, and predictors of fluoroquinolone resistance in multidrug resistant tuberculosis patients. Braz $\mathrm{J}$ Infect Dis [serial online] $2016 \mathrm{Jan}-\mathrm{Feb}$ [cited 2020 May 13]; 20(1):41-7. https://doi.org/10.1016/j. bjid.2015.09.011

9. Alene KA, Yi H, Viney K, McBryde ES, Yang K, Bai $L$, et al. Treatment outcomes of patients with multidrug-resistant and extensively drug resistant tuberculosis in Hunan Province, China. BMC Infect Dis [serial online] 2017 [cited 2020 May 14]; 17:573. https://doi.org/10.1186/s12879017-2662-8

10. Wahab F, Ashraf S, Khan N, Anwar R, Afridi MZ. Risk factors for multi-drug resistant tuberculosis in patients at tertiary care hospital, Peshawar. J Coll Physicians Surg Pak [cited 2020 May 14] 2009 Mar; 19(3):162-4. doi: 03.2009/JCPSP.162164

11. Akhtar AM, Arif MA, Kanwal S, Majeed S. Prevalence and drug resistance pattern of MDR TB in retreatment cases of Punjab, Pakistan. J Pak Med Assoc 2016 Aug; 66(8):989-93.

12. Javaid A, Hasan R, Zafar A, Chaudry MA, Qayyum $S$, Qadeer E, et al. Pattern of first- and second-line drug resistance among pulmonary tuberculosis retreatment cases in Pakistan. Int $\mathrm{J}$ Tuberc Lung Dis 2017 Mar 1; 21(3):303-8. https://doi. org/10.5588/ijtld.16.0444

13. WHO. TB disease burden: Companion handbook to the WHO guidelines for the programmatic management of drug-resistant tuberculosis. WHO, Geneva. WHO Document Production Services 2014.p.27-70.

14. Raosoft $\circledast$ sample size calculator. [accessed 2020 May 14]. Available at: http://www.raosoft.com/ samplesize.html 
15. Statistics Kingdom. Proportion confidence interval calculator [internet]. Statistics Kingdom; Melbourne, Australia 2007. [accessed 2020 May 07]. Available at: http://www.statskingdom. com/41_proportion_confidence_interval.html

16. Zar JH. Biostatistical Analysis. 5th ed. New York: Prentice-Hall, Inc.

17. Daniel WW. Biostatistics: A Foundation for Analysis in the Health Sciences. 7th ed. Singapore: John Wily; 2005.

18. Jeremy Stangroom. Chi-Square Test Calculator. Social Science Statistics. [accessed 2020 Apr 24]. Available at: https://www.socscistatistics. com/ tests/chisquare2/Default2.aspx

19. Statistics Kingdom. Chi-Square test calculator [internet]. Statistics Kingdom; Melbourne, Australia 2007. [accessed 2020 May 07]. Available at: https://www.statskingdom.com/310GoodnessChi.html

20. Shah S, Basharat A, Shah M, Marwat M, Billah M, Ali SM. Frequency, distribution and presentation $\mathrm{n}$ of hypocalcemia in B-thalassemia major. Gomal J Med Sci 2018; 16:2-8. https://doi.org/10.46903/ gjms/16.01.1446

21. Ghori MR, Khan H, Marwat M. Distribution of non-malignant hematological disorders by sex, age groups and type of disease based on bone marrow aspiration in population of Khyber Pa-khtunkhwa, Pakistan. Gomal J Med Sci 2019 Apr-Jun; 17 (2): 29-36. https://doi.org/10.46903/ gjms/17.02.1926

22. Butt M, Rehman MU, Khan AR, Abrar A. Frequency of triple-vessel coronary artery disease in adult type 2 diabetics versus non-diabetics in coronary artery disease population of Islamabad, Pakistan.
Gomal J Med Sci 2019 Apr-Jun; 17(2).37-41. https://doi.org/10.46903/gjms/17.02.2029

23. Ain N, Khan S, Marwat M, Khan N, Ahmad I, Ramzan F, et al. Frequency, distribution and determinants of hypertension in adult stroke population of D.I.Khan Division, Pakistan. Gomal J Med Sci 2019 Jul-Sep; 17 (3):81-9. https://doi. org/10.46903/gjms/17.03.2076

24. Akhtar A, Ali SM, Naqi SA, Aziz T, Shinwari W, Shah SFH. Efficacy of acetic acid versus ciprofloxacin ear drops in achieving dry ears in chronic suppurative otitis media in adult population of Islamabad, Pakistan: a non-randomized control trial. Gomal J Med Sci 2019 Jul-Sep; 17 (3): 90-94. https://doi.org/10.46903/gjms/17.03.2007

25. Marwat M, Ahmad I, Ashiq F, Ali S, Zamir S, Rehman MU, et al. Frequency, distribution and determinants of diabetes mellitus in adult acute coronary syndrome population of D.I.Khan Division, Pakistan. Gomal J Med Sci 2019 OctDec; 17 (4):131-43. https://doi.org/10.46903/ gjms/17.04.2106

26. Khan A, Khan MI, Ali MA, Hussain A, Ibrahim M, Muhammad T. Sex and age wise distribution, presentation and successful removal and mortality of foreign body aspiration using rigid bronchoscope in children of District D.I.Khan, Pakistan. Gomal J Med Sci 2020 Jan-Mar; 18 (1):311. https://doi.org/10.46903/gjms/18.01.1949

27. Khalid MK, Ahmad I, Khan MA, Sharjeel M, Irfan M. Marwat M. Distribution of pediatric cataracts by sex, age groups, laterality, type and presentation in population of D.I.Khan District, Pakistan. Gomal J Med Sci 2019 Oct-Dec; 17 (3):123-30. https:// doi.org/10.46903/gjms/17.04.2025

\section{CONFLICT OF INTEREST \\ Authors declare no conflict of interest. GRANT SUPPORT AND FINANCIAL DISCLOSURE None declared.}

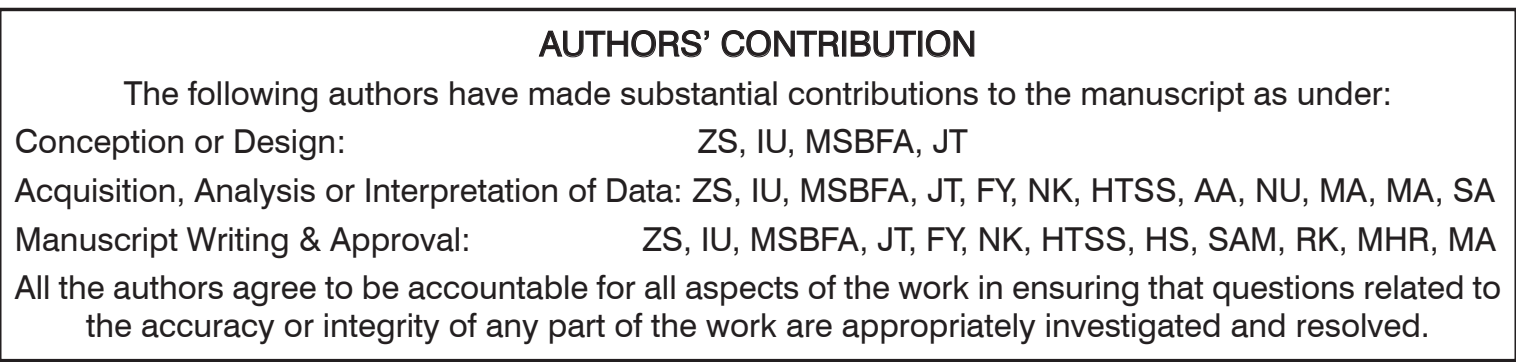

